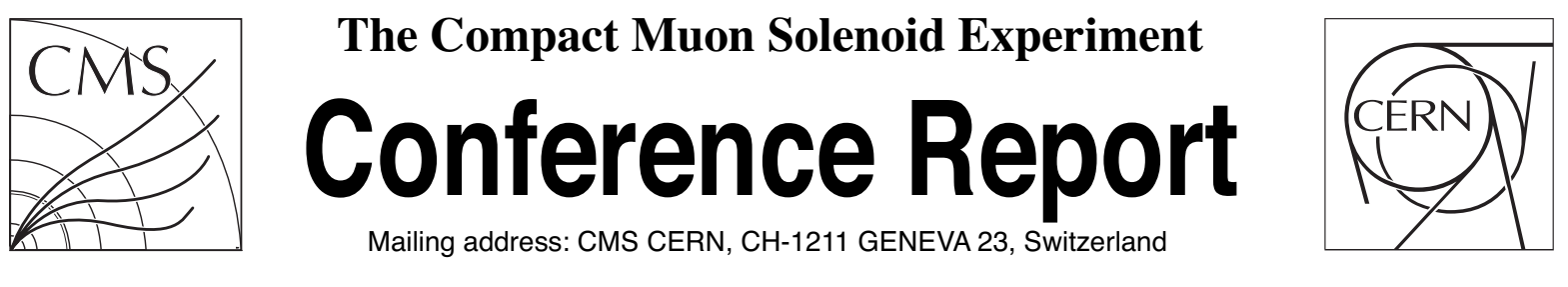

29 December 2015 (v2, 30 December 2015)

\title{
CMS bottomonia results from Run I
}

\author{
Mihee Jo for the CMS Collaboration
}

\begin{abstract}
Bottomonia are important probes of the quark-gluon plasma since they are produced at early times and propagate through the medium, mapping its evolution. The production cross section of the three $\mathrm{Y}$ states (1S, 2S, 3S) was measured separately using the Compact Muon Solenoid (CMS) experimental apparatus, in pp and $\mathrm{PbPb}$ collisions at $2.76 \mathrm{TeV}$. A strong suppression is observed in $\mathrm{PbPb}$ collisions, by up to a factor of 2 and 10 for the $\mathrm{Y}(1 \mathrm{~S})$ and $\mathrm{Y}(2 \mathrm{~S})$ respectively. The $\mathrm{Y}(3 \mathrm{~S})$ was not observed in $\mathrm{PbPb}$ collisions, being suppressed by more than 7 at the $95 \mathrm{pct}$ confidence level. This suppression was seen to also depend on centrality, but not significantly on transverse momentum or rapidity. A similar suppression pattern of the excited states (2S and $3 \mathrm{~S}$ ) as compared to the ground state (1S), though less pronounced than in $\mathrm{PbPb}$ data, was observed also when analyzing the $\mathrm{pPb}$ data at $5.02 \mathrm{TeV}$ center of mass collision energy. A surprising dependence of the excited over the ground state ratio, as a function of the global event activity, was also found in $\mathrm{pPb}$ data. The three states are also observed to be individually more produced in events with more activity, for the three collision systems. In this talk we will present the final CMS results on bottomonium production in the three collisions systems.
\end{abstract}




\title{
CMS bottomonia results from Run I
}

\author{
Mihee Jo for the CMS collaboration
}

Laboratoire Leprince-Ringuet, École polytechnique 91120 Palaiseau, France

\begin{abstract}
Bottomonia are important probes of the quark-gluon plasma since they are produced at early times and propagate through the medium, mapping its evolution. The production cross section of the three $\Upsilon$ states $(1 \mathrm{~S}, 2 \mathrm{~S}, 3 \mathrm{~S})$ is measured separately using the Compact Muon Solenoid (CMS) experimental apparatus, in pp and $\mathrm{PbPb}$ collisions at 2.76 TeV. A strong suppression is observed in $\mathrm{PbPb}$ collisions, by up to a factor of 2 and 10 for the $\Upsilon(1 \mathrm{~S})$ and $\Upsilon(2 \mathrm{~S})$ respectively. The $\Upsilon(3 \mathrm{~S})$ was not observed in $\mathrm{PbPb}$ collisions, being suppressed by more than 7 at the $95 \%$ confidence level. This suppression was seen to also depend on centrality, but not significantly on transverse momentum or rapidity. A similar suppression pattern of the excited states ( $2 \mathrm{~S}$ and $3 \mathrm{~S}$ ) as compared to the ground state (1S), though less pronounced than in $\mathrm{PbPb}$ data, was observed also when analyzing the $\mathrm{pPb}$ data at $5.02 \mathrm{TeV}$ center of mass collision energy. A surprising dependence of the excited over the ground state ratio, as a function of the global event activity, was also found in $\mathrm{pPb}$ data. The three states are also observed to be individually more produced in events with more activity, for the three collision systems. In this proceeding, the final CMS results on bottomonium production in the three collisions systems will be presented.
\end{abstract}

Keywords: Quarkonia, Bottomonia, Sequential suppression

\section{Introduction}

The quark-gluon plasma (QGP) can be created by the ultra-relativistic heavy-ion collisions, which is a deconfined state of quarks and gluons at a high medium temperature and density. One of the signatures of the QGP formation is the suppression of quarkonium states [1] when the binding energy of the quarkonium state is screened by the color charges of the neighboring partons. The dissociation temperature of the medium is expected to be dependent on the binding energy of quarkonia which results in the sequential suppression pattern of quarkonia. For instance, the $\Upsilon(1 \mathrm{~S})$ state has the strongest binding energy than any other quarkonia, hence $\Upsilon(1 \mathrm{~S})$ has the highest dissociation temperature and excited states, $\Upsilon(2 \mathrm{~S}, 3 \mathrm{~S})$, are dissociated at lower temperature.

However, besides color screening effect, there are other phenomena in heavy-ion collisions that could modify the quarkonia production. These include cold nuclear matter (CNM) effects such as modification of parton distribution function inside nucleus, Cronin effect and absorption by comoving particles, which do not require the formation of a QGP medium. Moreover, regeneration of initially uncorrelated quarkantiquark pairs would increase the production of quarkonia [2]. 
Bottomonia is a good probe among the other quarkonia which enables an easier interpretation of the final measurements. Bottomonia does not require a separation of prompt and non-prompt components nor cold nuclear matter effects. Also the regeneration of bottomonia are expected to be smaller than charmonia.

Proton-proton collisions are essential to understand elementary bottomonium production as a reference to $\mathrm{Pb}-\mathrm{Pb}$ collisions, and $\mathrm{Pb}$-proton collision can probe aforementioned cold nuclear matter effects. The Large Hadron Collider (LHC) provided both $\mathrm{PbPb}$ collisions in 2011 and proton-proton collisions in 2013 at $\sqrt{s_{N N}}=2.76 \mathrm{TeV}$, and the datasets collected by Compact Muon Solenoid (CMS) experiment correspond to an integrated luminosity of $166 \mu \mathrm{b}^{-1}$ and $5.4 \mathrm{pb}^{-1}$ datasets, respectively. The LHC also provided $\mathrm{Pb}-$ proton collisions in 2011 at $\sqrt{s_{N N}}=5.02 \mathrm{TeV}$ where a dataset of about $31 n b^{-1}$ by CMS.

$\Upsilon$ production in $\mathrm{Pb}$-proton collisions was measured as a function of event activity [3] for the first time. Thanks to the larger statistics on proton-proton reference dataset and improved $\mathrm{PbPb}$ reconstruction algorithm comparing to the previous $\Upsilon$ measurement that is published in Ref. [4], more precise mapping of the kinematics of the in-medium $\Upsilon$ suppression is obtained in Ref. [5]. In this proceeding, the latest $\Upsilon$ measurements in all three collision systems will be visited.

\section{Bottomonia in $\mathrm{pPb}$ collisions}

A ratio of bottomonia excited states to ground states is obtained from $\mathrm{pPb}$ and $\mathrm{pp}$ collisions over integrated event activity, and the double ratio is calculated by dividing the single ratios as described in Eq. 1. In this double ratio, acceptance and efficiency of $\Upsilon$ are canceled. The double ratios in $\mathrm{PbPb}$ collisions from Ref. [4] and in $\mathrm{pPb}$ collisions are presented on the left of Fig. 1. A large suppression of excited states compare to ground state in $\mathrm{PbPb}$ collisions is due to the presence of hot and dense medium, while a small suppression in $\mathrm{pPb}$ collisions suggests the final state effects for excited states are stronger than ground state in $\mathrm{pPb}$ collisions. On the center and right of Fig. 1, single ratios, $\Upsilon(\mathrm{nS}) / \Upsilon(1 \mathrm{~S})$, are drawn as a function of event activity. Event activity is determined with transverse energy deposited at forward hadronic calorimeter at $4<|\eta|<5.2$ for the figure in the center, and with number of charged tracks at $|\eta|<2.4$ for the figure in the right. The single ratios are found to decrease with increasing track multiplicity which lead to 2 possible scenarios: the multiplicity gives stronger impact on the more weakly bounded states, or $\Upsilon$ states are produced in systematically different way as a function of number of charged particles.

$$
\frac{\Upsilon(n S) /\left.\Upsilon(1 S)\right|_{p P b}}{\Upsilon(n S) /\left.\Upsilon(1 S)\right|_{p p}}=\frac{R_{p P b}(\Upsilon(n S))}{R_{p P b}(\Upsilon(1 S))}
$$
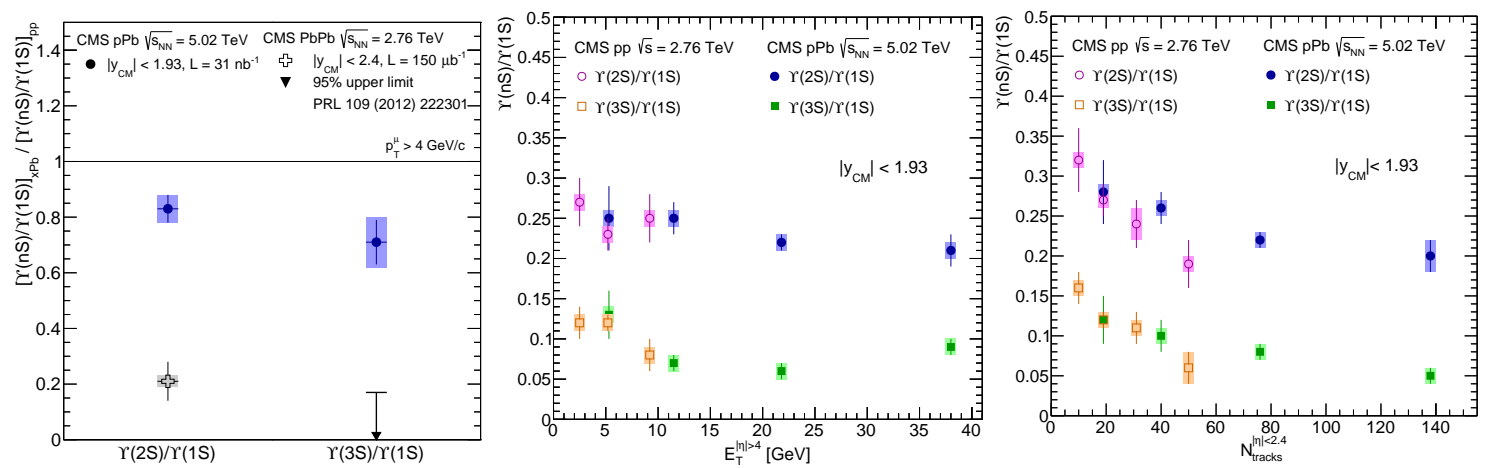

Fig. 1. On the left, double ratios of the $\Upsilon(\mathrm{nS})$ to the $\Upsilon(1 \mathrm{~S})$ in $\mathrm{pPb}$ collisions (circles) and the corresponding ratios in $\mathrm{PbPb}$ collisions (cross) is shown. On the center, single cross section ratios $\Upsilon(\mathrm{nS}) / \Upsilon(1 \mathrm{~S})$ for $\left|y_{\mathrm{CM}}\right|<1.93$ versus transverse energy measured in $4.0<|\eta|<5.2$ and on the right, number of charged tracks measured in $|\eta|<2.4$ are presented. All results are analyzed with pp and $\mathrm{PbPb}$ collisions at $\sqrt{s_{N N}}=2.76 \mathrm{TeV}$ (open symbols) and $\mathrm{pPb}$ collisions at $\sqrt{s_{N N}}=5.02 \mathrm{TeV}$ (closed symbols). Statistical (lines) and systematical (boxes) uncertainties are displayed. 


\section{Bottomonia in $\mathrm{PbPb}$ collisions}

For the most precise and detailed measurements of $\Upsilon$ in heavy-ion collisions from run 1 phase, the latest analysis has been optimized separately for ground state and excited states for signal extraction step. The latest pp data sample with an integrated luminosity of $5.4 \mathrm{pb}^{-1}$ allowed $\Upsilon(\mathrm{nS})$ differential cross section measurements as a function of $p_{T}$ and $|y|$ in Fig. 2. These are the first cross section measurements at $\sqrt{s}=$ $2.76 \mathrm{TeV}$ that provide an important input to production models. Furthermore, $\Upsilon(2 \mathrm{~S})$ spectrum is measured in $\mathrm{PbPb}$ collisions for the first time that are presented at the bottom of Fig. 2. Due to the lack of $\Upsilon(2 \mathrm{~S})$ statistics in $\mathrm{PbPb}$ dataset, wider bin width is used than that of pp dataset.
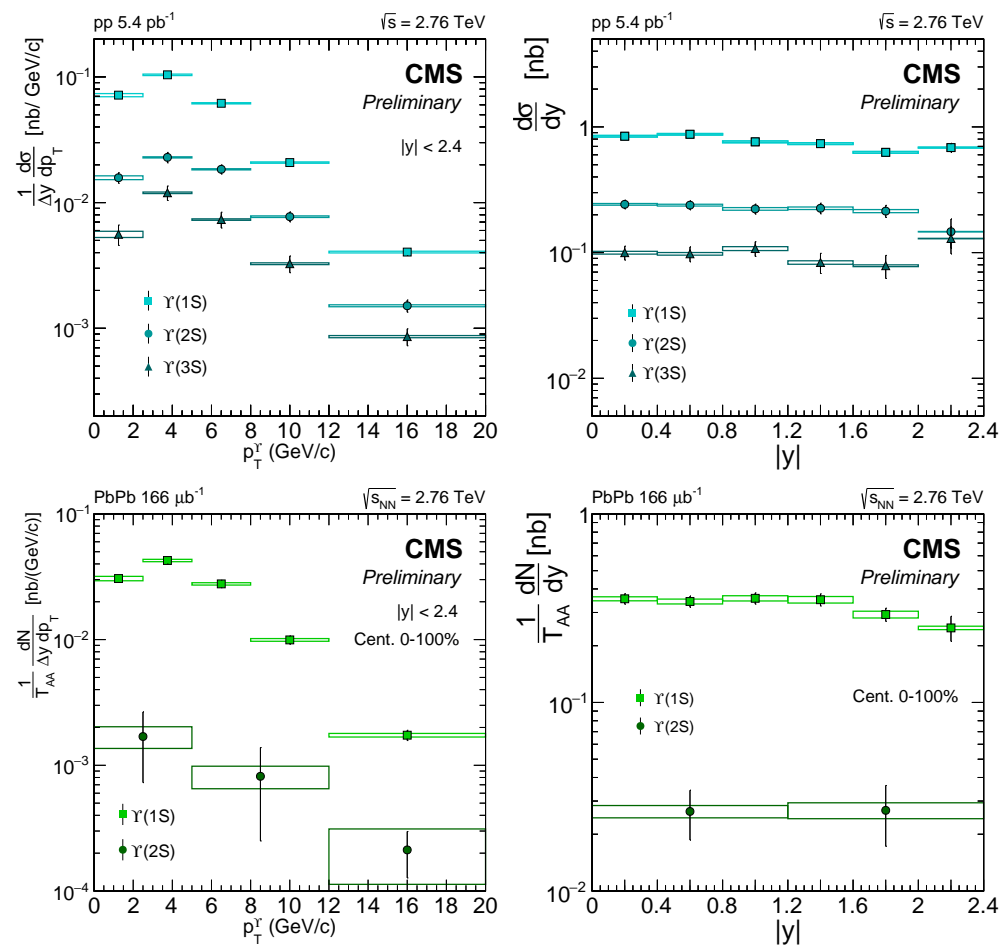

Fig. 2. The differential cross sections of the $\Upsilon(\mathrm{nS})$ as a function of $p_{T}$ (left) and $|y|$ (right) in pp (top) and $\mathrm{PbPb}$ (bottom) collisions at $\sqrt{s_{N N}}=2.76 \mathrm{TeV}$ are shown. Global uncertainties of $5 \%$ (top) and $10.5 \%$ (bottom) are not displayed.

Nuclear modification factors, $R_{A A}$, are obtained by dividing the corrected $\mathrm{PbPb}$ yields by the pp cross sections and $T A A$ values in Fig. 3 and they are compared to theoretical models and ALICE results, too. $\Upsilon(3 \mathrm{~S})$ is not observed in $\mathrm{PbPb}$ collisions, hence only an upper limit of the $R_{A A}$ is derived which is less than 0.14 at $95 \%$ C.L. integrated over the entire kinematic and centrality ranges. In centrality $0-100 \%$ case, $\Upsilon(1 \mathrm{~S}, 2 \mathrm{~S})$ are suppressed by a factor of 2 and 10, respectively. Strong suppression pattern as a function of centrality is observed for $\Upsilon(1 \mathrm{~S})$ while $\Upsilon(2 \mathrm{~S})$ is largely suppressed for all centrality. No pronounced $p_{T}$ nor $|y|$ dependent suppression is observed for both $\Upsilon(1 \mathrm{~S}, 2 \mathrm{~S})$ in the region of CMS measurement, $|y|<2.4$ and $p_{T}<20 \mathrm{GeV} / \mathrm{c}$.

On the top left side of the Fig. 3, the $R_{A A}$ of $\Upsilon(1 \mathrm{~S}, 2 \mathrm{~S})$ are well described by a model with potential-based non-relativistic effective field theory (pNRQCD) updated with anisotropic hydrodynamics and feed-down fractions [6]. On the top right side, cold nuclear matter effects and regeneration mechanisms in the QGP and in hadronization phases are accounted in a theoretical model [7] which has a good agreement with CMS measurements.

Theoretical models as a function of meson kinematics are presented on the bottom panel in Fig. 3. Model calculations are in good agreement with all experimental results with respect to $|y|$ in $|y|>2.4$ region. 
However, $\Upsilon(1 \mathrm{~S})$ is more suppressed at $y>2.4$ than mid-rapidity when extending the rapidity range of $\Upsilon(1 \mathrm{~S})$ $R_{A A}$ by combining results from ALICE experiment [8]. Measured $\Upsilon(1 \mathrm{~S}) R_{A A}$ and the model calculation are matched well while there is a little bit of tension on $\Upsilon(2 \mathrm{~S}) R_{A A}$.
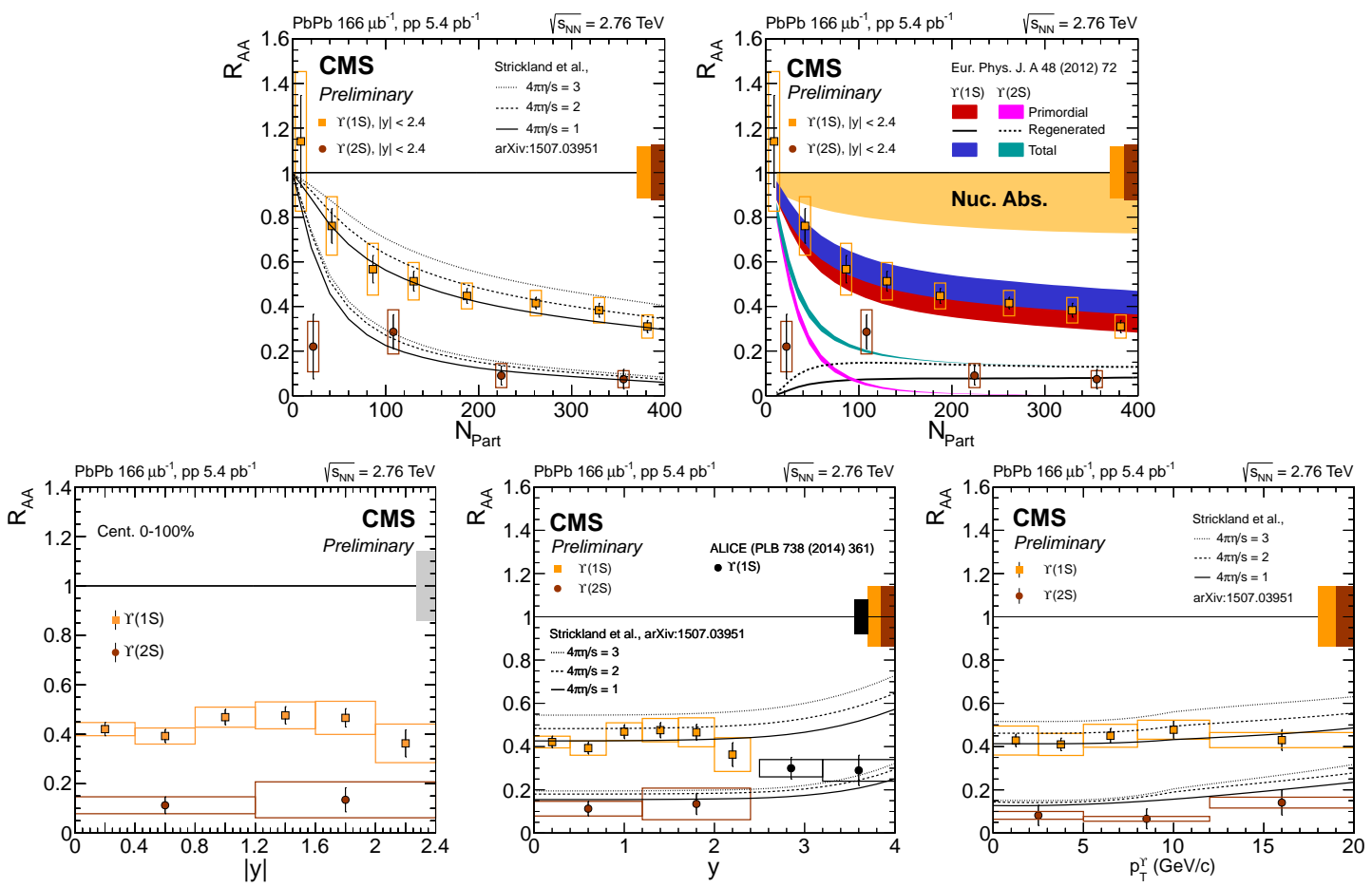

Fig. 3. The $R_{A A}$ of the $\Upsilon(1 \mathrm{~S}, 2 \mathrm{~S})$ are compared to theory predictions. Theory models as a function of $N_{\text {part }}$ from Ref. [6] (top, left) and from Ref. [7] (top, right). The $\Upsilon(1 \mathrm{~S}, 2 \mathrm{~S}) R_{A A}$ as a function of $|y|$ is measured by CMS (bottom, left) but $y$ is used for $\Upsilon$ (1S) measurement comparison to ALICE (bottom, center) [8]. Theory models from Ref. [6] is also compared to $R_{A A}$ with respect to $p_{T}$ (bottom, right) and $y$ (bottom, center) are compared to theory predictions from Ref. [6].

\section{Summary}

The $\Upsilon(1 \mathrm{~S}, 2 \mathrm{~S}, 3 \mathrm{~S})$ are measured in $\mathrm{pp}, \mathrm{PbPb}$ and $\mathrm{pPb}$ collisions at the center of energy 2.76 and 5.02 $\mathrm{TeV}$, respectively, during run 1 phase of the LHC. Cold nuclear matter effects on bottomonia are probed in $\mathrm{pPb}$ collisions that result a small amount of suppression on excited states comparing to ground state than $\mathrm{pp}$ collisions. Together with increasing single ratios of bottomonia with respect to charged track multiplicity, these results indicate that the final state effects in $\mathrm{pPb}$ collisions are stronger on excited states than ground states. $R_{A A}$ of bottomonia has been measured in $\mathrm{PbPb}$ collisions as a function of $N_{\text {part }}, p_{T}$ and $|y|$. Bottomonia are all suppressed comparing to pp collisions with no dependence on meson kinematics for $\Upsilon(1 \mathrm{~S}, 2 \mathrm{~S})$. Centrality dependent suppression is observed for $\Upsilon(1 S)$ while $\Upsilon(2 S)$ has a rather flat suppression pattern.

\section{References}

[1] T. Matsui and H. Satz, Phys. Lett. B 178, 416 (1986).

[2] A. Andronic et al, arXiv:1506.03981.

[3] CMS collaboration, J. High Energy Phys. 04 (2014) 103.

[4] CMS collaboration, Phys. Rev. Lett. 109 (2012) 222301.

[5] CMS collaboration, CMS-PAS-HIN-15-001.

[6] B. Krouppa, R. Ryblewski, M. Strickland, Phys. Rev. C 92, 061901 (2015).

[7] A. Emerick, X. Zhao, R. Rapp, Eur. Phys. J. A48 (2012) 72.

[8] ALICE collaboration, Phys. Lett. B 738, 361 (2014). 